\title{
Impressive near-infrared brightness and singlet oxygen generation from strategic lanthanide-porphyrin double-decker complexes in aqueous solution
}

\author{
Jing-Xiang Zhang $\mathbb{1}^{1,2}$, Wai-Lun Chan $\mathbb{D}^{1}$, Chen Xie ${ }^{1}$, Yan Zhou' ${ }^{1}$, Ho-Fai Chau ${ }^{3}$, Partha Maity $\mathbb{D}^{4}$, George T. Harrison ${ }^{4}$,

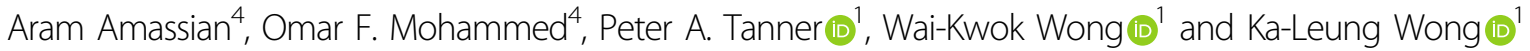

\begin{abstract}
Although lanthanide double-decker complexes with hetero-macrocyclic ligands as functional luminescent and magnetic materials have promising properties, their inferior water solubility has negated their biomedical applications. Herein, four water-soluble homoleptic lanthanide $(\mathbf{L n}=\mathbf{G d}, \mathbf{E r}$, $\mathbf{Y b}$ and $\mathbf{L a})$ sandwiches with diethylene-glycoldisubstituted porphyrins (DD) are reported, with their structures proven by both quantum chemical calculations and scanning tunneling microscopy. Our findings demonstrate that the near-infrared emission intensity and singlet oxygen $\left({ }^{1} \mathrm{O}_{2}\right)$ quantum yields of $\mathbf{Y b D D}$ and $\mathbf{G d D D}$ in aqueous media are higher than those of the reported capped lanthanide monoporphyrinato analogues, $\mathbf{Y b N}$ and $\mathbf{G d N}$; the brightness and luminescence lifetime in water of $\mathbf{Y b D D}$ are greater than those of $\mathbf{Y b N}$. This work provides a new dimension for the future design and development of molecular theranostics-based water-soluble double-decker lanthanide bisporphyrinates.
\end{abstract}

\section{Introduction}

Near-infrared (NIR) luminescent lanthanide materials have been widely utilized and increasingly researched in telecommunications engineering, laser technology, and biomedical science by virtue of their extraordinary photophysical properties ${ }^{1-5}$. However, challenges remain that lanthanides are intrinsically constrained by the Laporteforbidden $4 f-4 f$ transitions that render their direct excitation rather inefficient ${ }^{6,7}$. To circumvent this issue, $\pi$-conjugated hetero-macrocycles, such as porphyrins, possessing (i) high-absorption cross-sections, (ii) triplet states resonating well with lanthanide absorption bands,

\footnotetext{
Correspondence: Ka-Leung Wong (klwong@hkbu.edu.hk) or

Peter A. Tanner (peter.a.tanner@gmail.com) or

Wai-Kwok Wong (wkwong@hkbu.edu.hk)

'Department of Chemistry, Hong Kong Baptist University, Kowloon Tong, Hong Kong S.A.R., China

${ }^{2}$ Hanshan Normal University, Chaozhou, Guangdong Province, China Full list of author information is available at the end of the article.

These authors contributed equally: Jing-Xiang Zhang, Wai-Lun Chan, Chen Xie
}

and (iii) four "hard" nitrogen donor atoms matching "hard" lanthanides, have become promising antenna in use for optimal energy sensitization and protective coordination $^{8-14}$. Sandwich-type lanthanide-porphyrin complexes can afford more preferable or even surprising emission results, given that double-decker lanthanide complexes have recently spanned the fields of electrochromic/optoelectronic devices, photovoltaic cells, singlemolecule magnets, and even molecular rotors-though with few bio-related counterparts ${ }^{15-18}$. Despite their wellcharacterized, long-lived NIR emission, and ${ }^{1} \mathrm{O}_{2}$ generation, most lanthanide-macrocycle complexes suffer from inferior water solubility that considerably hampers their further development in biomedical fields ${ }^{17,18}$. Tarakanova et al. performed the first comprehensive study on doubledecker lanthanide complexes and investigated their interaction with water. Unfortunately, only one incorporated water molecule was considered and only intramolecular hydrogen bonding was examined, without the

\section{(c) The Author(s) 2019}

(c) (i) Open Access This article is licensed under a Creative Commons Attribution 4.0 International License, which permits use, sharing, adaptation, distribution and reproduction cc) in any medium or format, as long as you give appropriate credit to the original author(s) and the source, provide a link to the Creative Commons license, and indicate if changes were made. The images or other third party material in this article are included in the article's Creative Commons license, unless indicated otherwise in a credit line to the material. If material is not included in the article's Creative Commons license and your intended use is not permitted by statutory regulation or exceeds the permitted use, you will need to obtain permission directly from the copyright holder. To view a copy of this license, visit http://creativecommons.org/licenses/by/4.0/. 
description of an aqueous solution ${ }^{19}$. Recently, watersoluble gadolinium-porphyrin complexes were reported by Zang et al. ${ }^{20}$. Their complexes have two porphyrin rings but do not form a sandwich structure. Therefore, their recorded molar extinction coefficients and singlet oxygen quantum yield in water were much lower than those of our Gd-analogues, although these two series of complexes both have similar two porphyrin rings as antenna chromophores. The porphyrin structure is rigid and its excited energy can be nonradiatively transferred to an acceptor ${ }^{21}$. Thus, we recently focused on and have already reported three water-soluble (up to $1 \mu \mathrm{M}$ ), polyethylene glycol (PEG) chain-conjugated, capped lanthanide monoporphyrinates of (i) organelle specificity, $\mathbf{Y} \mathbf{b R h} \mathbf{B}^{22}$, (ii) tumor selectivity, $\mathbf{Y} \mathbf{b} \mathbf{N}^{23}$, and (iii) photodynamic therapy, $\mathbf{G d N}^{24}$. Herein, we introduce four water-soluble porphyrin-based lanthanide double-decker complexes (LnDD, where $\mathrm{Ln}=\mathrm{La}, \mathrm{Er}, \mathrm{Gd}$ and Yb, Fig. 1a) with remarkable NIR photophysical properties in aqueous solution. Upon the strategic installation of two optimally short hydrophilic methylated diethylene glycol (DEG) chains on the tailor porphyrin Por(2DEG) for sandwich lanthanide complexation, YbDD exhibited improved NIR luminescence quantum yield and lifetime in water and outperformed previously reported $\mathbf{Y b N}$. The singlet oxygen generation efficiency in terms of quantum yield $\left(\Phi_{\mathrm{O} 2}\right)$ of GdDD was measured to be slightly higher than that of GdN. Our findings substantiate the hypotheses that the double-decker complexation between porphyrins can (i) facilitate better lanthanide sensitization in the presence of two antenna chromophores rather than one and (ii) minimize the innersphere quenching effect by lowering the number of bound water molecules under the macrocyclic sandwich design. This work provides unique results for the photophysical data of LnDD in aqueous media and more importantly, a new dimension for the future design and development of molecular theranostics-based watersoluble double-decker lanthanide bisporphyrinates. Structural elucidation in this study was performed using various techniques, as described herein, since it is difficult to prepare single crystals suitable for X-ray analysis.

\section{Results}

Structural characterization by calculation

The porphyrin dianion unit is planar with perpendicular aromatic rings (Supplementary Fig. S31). The structures of a
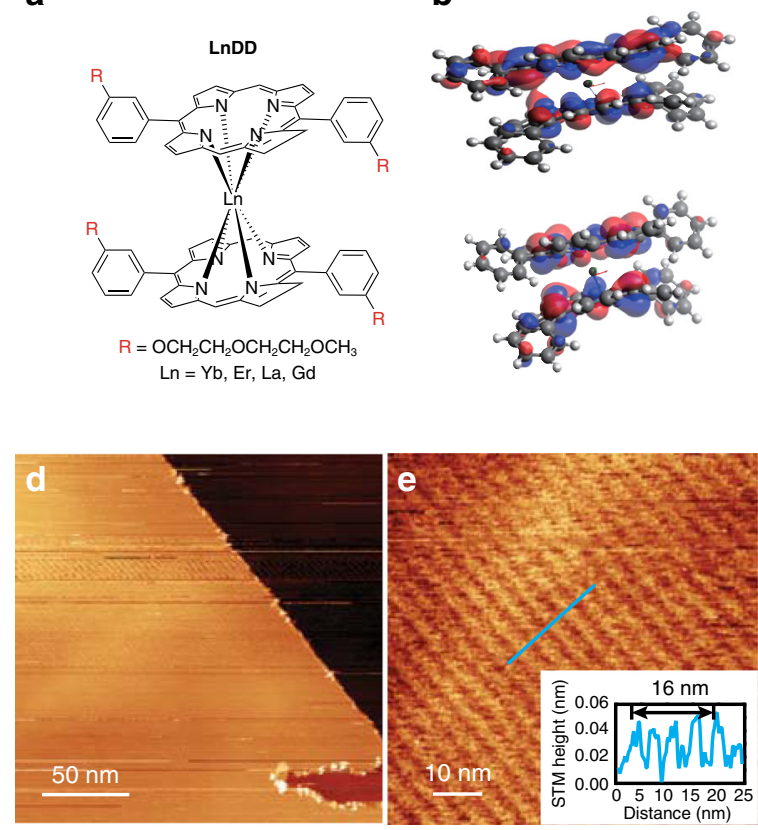

C
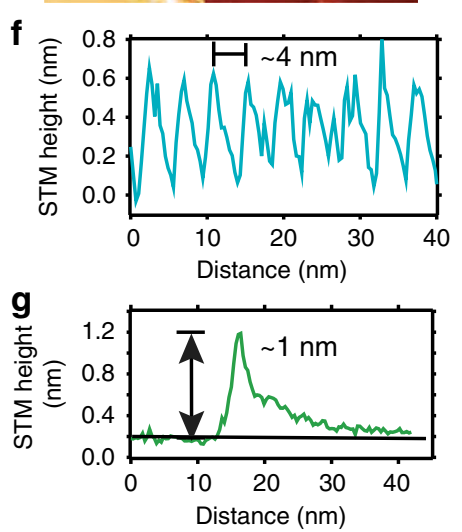

Fig. 1 Structure characterization. a Molecular structures of water-soluble lanthanide porphyrin complexes: use of porphyrins with double-decker forms with DEG-chain-conjugated porphyrins. b Calculated LUMO (top) and HOMO (bottom) by applying density functional theory (sidechains not included); STM images for YbDD drop-casting onto HOPG: c toluene $1 \mathrm{mmol} 5 \mu \mathrm{L}, 140 \times 140 \mathrm{~nm}^{2},-1.5 \mathrm{~V}$ sample bias, $50 \mathrm{pA}$, (inset) blue box: a highmagnification image, cyan and green lines denote the height line profiles (Fig. 1f, g); $\mathbf{d}$ chloroform $1 \mathrm{mmol} 25 \mu \mathrm{L} 270 \times 270 \mathrm{~nm}^{2}$ image, $+2 \mathrm{~V}$ sample bias, $100 \mathrm{pA}$, e $75 \times 75 \mathrm{~nm}^{2}$, image $+2 \mathrm{~V}$ sample bias, $100 \mathrm{pA}$, (inset) blue height profile across rows. $\mathbf{f}$ Line profile from Fig. 1c along the row of features. $\mathbf{g}$ Line profile across the HOPG step edge, the height of the feature $\sim 1 \mathrm{~nm}$ (bottom terrace) is indicated 
lanthanide double-decker porphyrins have been previously discussed in the literature ${ }^{25,26}$. The schematic structure of the double-decker complexes is depicted in Fig. 1a. The skeleton structure, without DEG sidechains and with or without a negative charge, was optimized by calculation (refer to Section 5, SI) because the total charge depends upon the $\mathrm{pH}$ and solvent. The structure optimization of YbDD and $[\mathrm{YbDD}]^{-}$using MOPAC ${ }^{27}$ in the LUMPAC 1.3.0 28,29 suite of programs is shown in Supplementary Fig. S32a, b, and is similar to that using the ORCA ${ }^{30}$ program (Supplementary Fig. S33) and Firefly $\mathrm{QC}^{31}$ package (Supplementary Figs. S35 and S37), which is partially based upon the GAMESS (US) ${ }^{32}$ source code. The porphyrin ring system is no longer planar due to (i) the cation- $\pi$ attractive forces and (ii) $\pi-\pi$ repulsive forces. The $\mathrm{N}-\mathrm{N}$ distance from the bottom to the top of the double decker is comparable with the distances within each sandwich layer. The structure was also optimized for the AlDD system (Supplementary Fig. S36) and shows six short Al-N bonds and two longer bonds. These bonds give rise to a distorted structure. The calculated highest occupied molecular orbital/lowest unoccupied molecular orbital (HOMO/LUMO) are also given for LnDD in Fig. 1b and Supplementary Fig. S34 (DEG chains are omitted for clarity).

\section{Structural characterization by scanning tunneling microscopy}

Scanning tunneling microscopy (STM) is an advanced technique that can be used for probing molecular assemblies on an individual molecule basis. The study of porphyrins assembly and structure at the vacuum and liquid interface on surfaces is relatively advanced ${ }^{33}$. In particular, several studies have been undertaken on double-decker structured molecules ${ }^{34,35}$. YbDD was deposited on a clean highly oriented pyrolytic graphite (HOPG) (0001) surface by placing a drop of dilute solution and evaporating at room temperature. The molecules formed self-assembled motifs without further treatment through surface adsorption and diffusion ${ }^{33}$. The STM topographic image in Fig. 1c shows a highmagnification image of a region of a drop-cast surface with additional features decorating the step edges. As shown in the zoom inset, these form a $\sim 4 \mathrm{~nm}$ periodic row of separation, and an apparent height of $\sim 1 \mathrm{~nm}$ (line profile Fig. $1 \mathrm{~g}$ ) is present. This height, which was recorded at $-1.5 \mathrm{~V}$ filled state, is strongly influenced by the electronic effects of both the tip apex and molecular surface junctions ${ }^{36,37}$. In a different trial with YbDD, a close-packed arrangement was observed and is shown in Fig. 1d, e. This ordered arrangement is long-ranged $\sim 100 \mathrm{~nm}$ and aligned parallel to the HOPG step edge direction. The features also show a separation of $\sim 4 \mathrm{~nm}$ in the direction perpendicular to the step direction (parallel unresolved) as indicated by the height line profile in Fig. 1e inset. Therefore, a templating effect originating at the step is suggested. The overall behavior of the drop-cast double-decker YbDD on HOPG is in line with previous studies of double-decker motifs and porphyrin ligands, with a favorable interaction and ability to spontaneously form a periodic assembly. The large $\sim 4 \mathrm{~nm}$ spacing between resolvable features is consistent with literature accounts of similarly structured molecules with the spacing correlated to alkyl chain length $^{34,38}$ with individual molecules packing face-on with the oxy-alkyl chain $\mathrm{R}$ groups having a favorable arrangement on the HOPG surface, leading to the observed spacing. We attribute the observed features, rows and protrusions (Fig. 1c, e) to single molecules with further work underway to resolve the exact inner-molecular structure.

\section{Structural characterization by nuclear magnetic resonance}

The synthesis and characterization of the double-decker porphyrinate lanthanide complexes with $\mathrm{Ln}=\mathrm{La}, \mathrm{Er}, \mathrm{Gd}$, and $\mathrm{Yb}$ trivalent ions are shown in Supplementary Scheme S1, Supplementary Figs. S1-S10 and Supplementary Table S1. Due to the paramagnetic properties of the latter three lanthanide ion complexes, LaDD was synthesized as the analogue for nuclear magnetic resonance (NMR) analysis. Upon the addition of hydrazine hydrate, a well-resolved LaDD NMR spectrum could be obtained (Fig. 2) because hydrazine hydrate served as a reducing agent and assisted the formation of monoanionic diamagnetic complexes ${ }^{9}$. The protons of the single ligand Por(2DEG) can be categorized into peripheral and internal. The peripheral aromatic protons are typically located approximately at $6.5-10.0 \mathrm{ppm}$, while the DEG sidechain aliphatic protons normally lie within the range of $1.5-4.0 \mathrm{ppm}$. The peak of the hydrazine hydrate mixed with DMSO- $d_{6}$ is observed at $2.6 \mathrm{ppm}$. The ring current effect strongly shifts the two internal protons on the porphyrin upfield to $-3.2 \mathrm{ppm}$. The disappearance of internal $\mathrm{N}-\mathrm{H}$ peaks and the proton shifting can then serve as an indication of metallization with the lanthanide ion. No signal is observed in the negative range (equated to internal $\mathrm{N}-\mathrm{H}$ protons) in the spectrum of LaDD, while all peaks are subjected to upfield shifting due to the anisotropy of the $f$-metal ion as well as the impact of lanthanide-induced shifts ${ }^{10}$. It is noted that the theoretically most possible supramolecular trimers or even multiple aggregate structures can also give rise to similar NMR spectra, but the high-resolution mass spectra (HRMS) and STM images corroborate the double-decker structure of LaDD (and thus the LnDD series) unambiguously (Supplementary Fig. S6).

\section{Photophysical studies and brightness}

Photophysical properties of $\mathrm{LnDD}(\mathrm{Ln}=\mathrm{Yb}, \mathrm{Er}, \mathrm{Gd}, \mathrm{La})$ have been measured (Supplementary Figs. S20-S24) and 


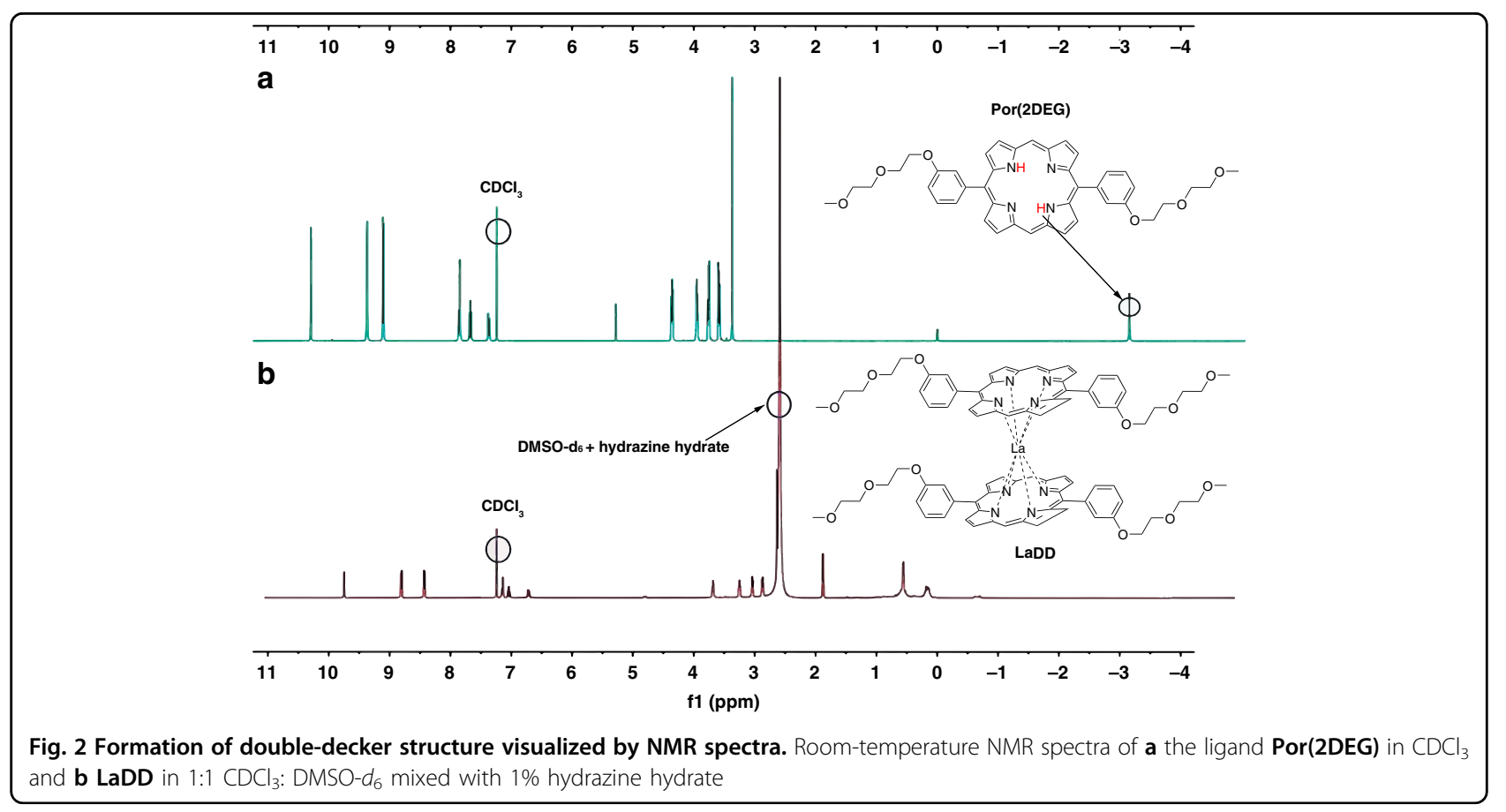

summarized in Supplementary Table S3. Upon photoexcitation at $425 \mathrm{~nm}$ (representing the strongest absorption band, the B or Soret band, Fig. 3a, black experimental spectrum), YbDD showcases superior photophysical performance compared with the monoporphyrinato counterpart/analogue $\mathbf{Y b N}$ (serving as the control) under various solvent systems (Supplementary Figs. S11-14 and Supplementary Table S2). The NIR quantum yields of YbDD were measured by comparison with the standard $\operatorname{YbTPP}(\mathbf{T p})$, which was reported as $3.2 \%$ in dichloromethane with the same excitation wavelength of $425 \mathrm{~nm}^{39}$. The NIR emission quantum yields of YbDD in toluene (water) were recorded as $3.5 \%(2.8 \%)$, while those of $\mathbf{Y b N}$ in these solvents were $2.8 \%$ (2.7\%), shown in Table 1. To explain these results, firstly, YbDD, which has two antenna ligand groups, should transcend $\mathbf{Y b N}$, which has only one. As shown in Fig. 3b, the emission spectrum of YbDD comprises several parts: the porphyrin ligand visible-NIR emission and the $\mathrm{Yb}^{3+}$ $\left({ }^{2} \mathrm{~F}_{5 / 2} \rightarrow{ }^{2} \mathrm{~F}_{7 / 2}\right)$ NIR emission, which has an equal peak height in this figure. The peaks at 647 and $699 \mathrm{~nm}$ represent the porphyrin fluorescence from the Q-band singlet nominally labeled $S_{1}$. The $S_{2}$ singlet (B-band) emission is also observed at a much weaker intensity and at shorter wavelengths (not shown). From the comparison with the low temperature $77 \mathrm{~K}$ emission spectrum of YbDD (Supplementary Fig. S19), the hot emission bands 1, 2, and 3 in Fig. $3 \mathrm{~b}$ may correspond to the transitions from the three excited states of ${ }^{2} \mathrm{~F}_{5 / 2}$, and the energy intervals between band 3 $\left(975 \mathrm{~nm} ; 10,260 \mathrm{~cm}^{-1}\right)$ and bands $4-6$ in Supplementary Fig. S19 can identify the three levels above the ground state energy of ${ }^{2} \mathrm{~F}_{7 / 2}$. The energy transfer from the porphyrin ligands to the $\mathrm{Yb}^{3+}$ ion is observed to be efficient because the metal ion is not excited by $425 \mathrm{~nm}$ radiation in the absence of an antenna. However, the presence of both ligand fluorescence and lanthanide emission at room temperature suggests that the energy transfer rate from the porphyrin to $\mathrm{Yb}^{3+}$ is similar to the nanosecond regime. The lower emission quantum yield of YbDD in water than that in toluene is attributable to the quenching by highfrequency $\mathrm{O}-\mathrm{H}$ vibrations. The trivalent lanthanide ions belong to the hard Lewis acid category with the coordination number of up to 8-12 so that under saturation of the lanthanides' inner coordination sphere by ligands offers vacancies for solvent molecule coordination ${ }^{35}$. The $\mathbf{Y b N}$ system was confirmed to have unsaturated sevencoordinated $\mathrm{Yb}^{3+}$ : four $\mathrm{N}$ from the porphyrin ring and three $\mathrm{O}$ from the Kläui $\left[\left(\eta^{5}-\mathrm{C}_{5} \mathrm{H}_{5}\right) \mathrm{Co}\left\{(\mathrm{MeO})_{2} \mathrm{P}=\mathrm{O}_{3}\right]^{-}\right.$ anion capped oxygen atoms. To shield the $\mathrm{Yb}^{3+}$ ion in an aqueous environment and suppress luminescence quenching, the double-decker complexation strategy in YbDD fulfills the eight-coordination number requirement.

Brightness is the product of quantum yield and molar attenuation coefficient ${ }^{40,41}$, and demonstrates the radiant energy emitted per frequency interval unit area per solid angle. For bioimaging purposes where low dosage is preferred because of adverse effects, the brightness is a more superior indicator of applicability than quantum yield, since, with higher brightness, low-abundance fluorescent compounds are detected more easily. The brightness of $\mathbf{Y b D D}$ exceeds that of $\mathbf{Y b N}$ by a factor of 

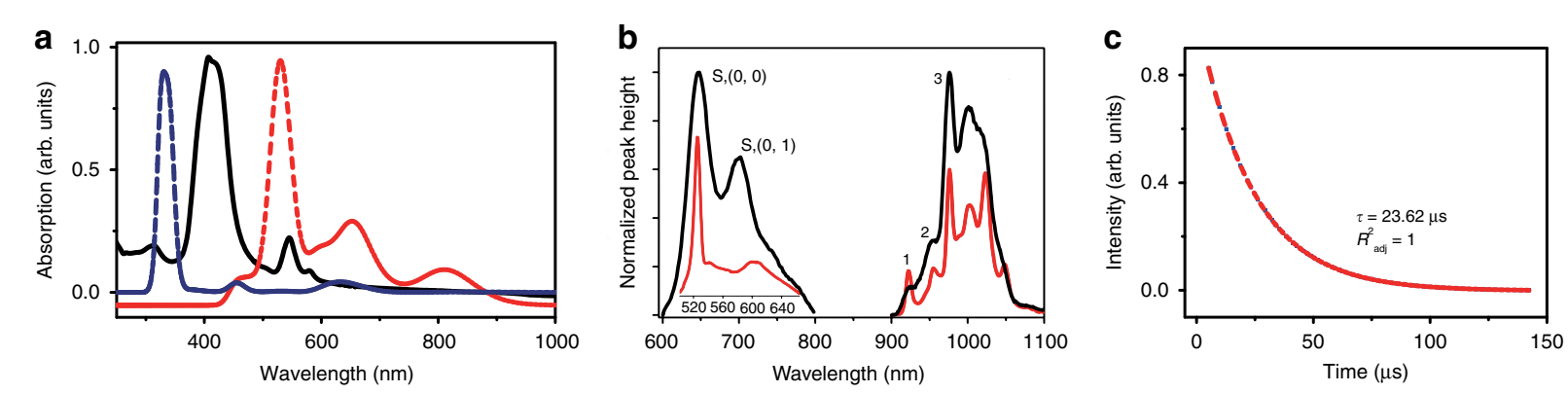

Fig. 3 Photophysical properties of the Yb(III) complexes. a Room temperature absorption spectrum of YbDD (black solid line) and calculated spectra using half-height widths of $1500 \mathrm{~cm}^{-1}$ using LUMPAC (blue dashed line) and ORCA (red dashed line) programs (refer to Supplymental Information Section 4). b Comparison of the emission bands of $\mathbf{Y b N}$ (black) and $\mathbf{Y b D D}$ (red) at $298 \mathrm{~K}$ in aqueous solution $\left(\lambda_{\mathrm{ex}}=425 \mathrm{~nm}\right)$. $\mathbf{c}$ Decay curve of $\mathrm{Yb}^{3+}$ emission in Fig. 3b (blue points) and fitted curve (red dashed line) using a monoexponential function

Table 1 Luminescence lifetime $(\tau)$, quantum yield $(\Phi)$ and brightness (BR) of $\mathrm{YbDD}$ and $\mathrm{YbN}$ in toluene or water

\begin{tabular}{llllll}
\hline & $\begin{array}{l}\boldsymbol{\tau}(\mu \mathrm{s})^{\mathbf{a}} \\
\text { Toluene }\end{array}$ & $\begin{array}{l}\boldsymbol{\tau}(\boldsymbol{\mu} \mathbf{s})^{\mathbf{a}} \\
\mathbf{H}_{\mathbf{2}} \mathbf{O}\end{array}$ & $\begin{array}{l}\boldsymbol{\Phi}(\%)^{\mathbf{b}} \\
\text { Toluene }\end{array}$ & $\begin{array}{l}\boldsymbol{\Phi}(\%)^{\mathbf{b}} \\
\mathbf{H}_{\mathbf{2}} \mathbf{O}\end{array}$ & $\begin{array}{l}\mathrm{BR}^{\mathbf{c}} \\
\mathbf{H}_{\mathbf{2}} \mathbf{O}\end{array}$ \\
\hline YbDD & 28.19 & 23.62 & 3.5 & 2.8 & 2540 \\
YbN & 23.02 & 12.04 & 2.8 & 2.7 & 1850 \\
\hline
\end{tabular}

aDetermined from the emission decay curve monitored at $\lambda_{\mathrm{em}}=978 \mathrm{~nm}$ $\left({ }^{2} \mathrm{~F}_{5 / 2} \rightarrow{ }^{2} \mathrm{~F}_{7 / 2}\right)$ with $\lambda_{\mathrm{ex}}=425 \mathrm{~nm}$ (Conc.: $1 \mu \mathrm{M}$ )

${ }^{\mathrm{b}}$ The relative quantum yields of the $\mathrm{Yb}^{3+}$ emission $\left(\lambda_{\mathrm{ex}}=425 \mathrm{~nm}\right)$ from the two $\mathrm{Yb}^{3+}$ complexes in toluene and $\mathrm{H}_{2} \mathrm{O}$ were obtained by comparison with the standard YbTPP(Tp)

${ }^{\mathrm{C}}$ Calculated from $\mathrm{BR}=$ attenuation coefficient $\times$ quantum yield; molar attenuation coefficients were obtained from the absorption spectra in water at $425 \mathrm{~nm}$ by applying the Beer-Lambert Law

1.37 (Table 1). The NIR ${ }^{2} \mathrm{~F}_{5 / 2} \rightarrow{ }^{2} \mathrm{~F}_{7 / 2}$ emission lifetimes of YbDD and $\mathbf{Y b N}$ were determined to be $23.6 \mu$ s in water (Fig. 3c and Supplementary Fig. S17, S18) and $28.2 \mu$ s in toluene, which are both higher than the values for $\mathbf{Y b N}$ (Table 1). YbDD shows a longer ${ }^{2} \mathrm{~F}_{5 / 2} \rightarrow{ }^{2} \mathrm{~F}_{7 / 2}$ lifetime, which mainly results from its higher symmetry than $\mathbf{Y b N}$. With a more symmetric structure, the $f-f$ transition mechanism is of less forced electric dipole character and more vibronic, and the lifetime for a specific transition is longer ${ }^{42}$. This trend is consistent with the measured NIR emission quantum yields in water and toluene. It is worth noting that most porphyrin-based NIR dyes for biological applications have little emission in the NIR-II biological window because no metal ion is coordinated. Furthermore, the maximal absorption peaks of these dyes are usually located only from 650 to $800 \mathrm{~nm}^{43}$. Both of these reasons limit the application prospects. One commercially available NIR-II dye (NIR-II dye \#900883, Sigma-Aldrich) has a similar emission peak located at $1050 \mathrm{~nm}$, which is the same as that of YbDD, but its NIR emission quantum yield is $\sim 2 \%$, which is lower than that of YbDD. The impressive NIR emission quantum yields and long NIR emission lifetime of YbDD in aqueous solution, together with its hydrophilic property, hold tremendous promise as a (NIR) bioimaging probe.

\section{Singlet-oxygen generation}

As a cross-system validation, the singlet oxygen quantum yield of GdDD was also examined in chloroform by comparison with the spectrum of the reference compound $\mathrm{H}_{2}$ TPP $\left(\Phi_{\Delta}=55 \%\right.$ in $\left.\mathrm{CHCl}_{3}\right)$. A new-generation anticancer agent $\mathbf{G d N}$, which consists of only one porphyrin ring, with high-singlet oxygen quantum yield was selected to serve as a comparison. The near-infrared ${ }^{1} \mathrm{O}_{2}$ phosphorescence spectra of GdDD, GdN and the reference are shown in Fig. 4a. From these spectra, the singlet oxygen quantum yields of GdDD and GdN were measured at $66 \%$ and $51 \%$, respectively. The singlet-oxygen quantum yield was also evaluated in aqueous solution with a PBS buffer using rose bengal (RB) as the standard by absorption changes of the decomposition of 9,10anthracenediyl-bis (methylene) dimalonic acid (ABDA) at $402 \mathrm{~nm}$ (Supplementary Figs. S15 and S16). The values of $\Phi_{\Delta}$ were determined as $46 \%$ for GdDD and $42 \%$ for $\mathbf{G d N}$. Hence GdDD displayed superior singlet oxygen generation in both organic and aqueous media. The comparison with two U.S. Food \& Drug Administration approved PDT agents, porfimer sodium (Photofrin $\left.{ }^{\circledR}\right)$ and 5aminolevulinic acid $\left(\operatorname{Levulan}^{\circledast}\right)$ was made. Although GdDD shows lower-singlet oxygen quantum yield ( $46 \%$ in aqueous solution, Photofrin ${ }^{\circledast}: 89 \%$; Levulan ${ }^{\oplus}: 56 \%$ ), it has a much higher maximal absorptivity (GdDD: $223,872 \mathrm{M}^{-1} \mathrm{~cm}^{-1} @ 412 \mathrm{~nm}$, and $52480 \mathrm{M}^{-1} \mathrm{~cm}^{-1}$ $@ 580 \mathrm{~nm}$ ) than these two commercial photosensitizers (Photofrin ${ }^{\circledR}: \quad 3000 \mathrm{M}^{-1} \mathrm{~cm}^{-1} @ 632 \mathrm{~nm}$; Levulan ${ }^{\circledR}$ : $\left.5000 \mathrm{M}^{-1} \mathrm{~cm}^{-1} @ 632 \mathrm{~nm}\right)^{44}$. With a double-decker porphyrinato structure and the resulting high molar extinction coefficient values, GdDD shows great applicability in photodynamic effects, which is also consistent with the 

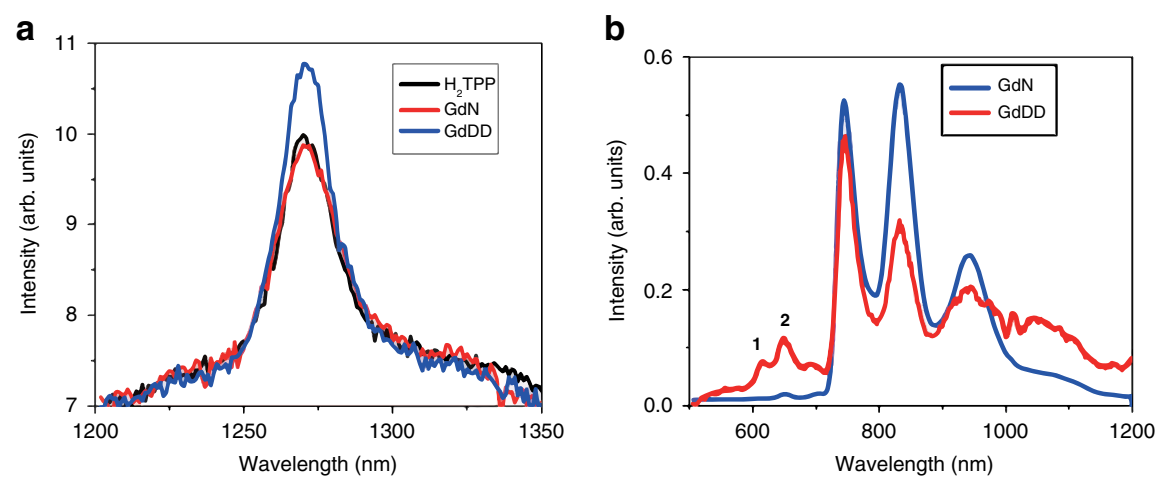

Fig. 4 Emission spectra of the $\mathrm{Gd}$ (III) complexes. a The near-infrared ${ }^{1} \mathrm{O}_{2}$ phosphorescence spectrum sensitized by GdDD, GdN, and the standard tetraphenylporphyrin $\mathrm{H}_{2}$ TPP (in $\mathrm{CHCl}_{3}$. Absorbance $=0.05$ at the excitation wavelength of $425 \mathrm{~nm}$ ). $\mathbf{b}$ The $77 \mathrm{~K}$ phosphorescence spectrum of $\mathbf{G d D D}$ and $\mathbf{G d N}$ in $\mathrm{MeOH}$ (Conc.: $10 \mu \mathrm{M}, \lambda_{\mathrm{ex}}=425 \mathrm{~nm}$ )

high brightness of YbDD. In vitro experiments have also been performed to practically compare photodynamic therapeutic efficiency in different cell lines, which also suggest GdDD as a potential PDT agent (Supplementary Figs. S25-S30 and Supplementary Table S4).

The energy gap between the antenna donor state and the lanthanide ion plays a crucial role in the energy transfer efficiency. The lowest triplet state of the lanthanide double-decker complex was determined experimentally from phosphorescence. The $77 \mathrm{~K}$ phosphorescence spectra of GdDD and GdN are shown in Fig. 4b. The zero-phonon lines are at a very similar wavelength $\left(\sim 745 \mathrm{~nm}: 13405 \mathrm{~cm}^{-1}\right)$, and the prominent vibrational progression in the ring carbon-nitrogen stretching mode of $1410 \mathrm{~cm}^{-1}$ is at a lower energy. The triplet energy level is therefore located at $2610 \mathrm{~cm}^{-1}$ above the highest ${ }^{2} \mathrm{~F}_{5 / 2}$ level of $\mathrm{Yb}^{3+}$ in YbDD. The optimum energy gap has been given as between 2000 and $5000 \mathrm{~cm}^{-1}$ to eradicate back energy transfer ${ }^{45,46}$. The weak features marked 1 and 2 in Fig. $4 \mathrm{~b}$ correspond to the singlet fluorescence bands $S_{1}(0,0)$ and $S_{1}(0,1)$, as in Fig. 3b for YbDD at $298 \mathrm{~K}$. The triplet state lifetimes of GdDD and $\mathbf{G d N}$ at $77 \mathrm{~K}$ were measured as $0.21 \pm 0.03$ and $0.14 \pm$ $0.02 \mathrm{~ms}$, respectively.

\section{Absorption spectrum and transient absorption spectroscopy}

Previous calculations of the energy levels of doubledecker complexes have shown poor agreement with experiments ${ }^{47,48}$. Herein, the absorption spectrum was modeled from the optimized structure by two programs. First, an excited states calculation was performed using the RM1 semiempirical quantum chemistry method using the LUMPAC suite of programs ${ }^{28,29}$, and the calculated result is shown as the dashed blue line in Fig. 3a. The strong singlet-singlet transition is located at $346 \mathrm{~nm}$. In the alternative calculation using $\mathrm{ORCA}^{30}$, this feature is shifted to lower energy at $530 \mathrm{~nm}$ (red dashed line, Fig. 3a).

Transient absorption (TA) spectroscopy, as twodimensional spectroscopy, was used to investigate both the spectral and temporal properties of the samples. The femtosecond (fs) TA spectra at different delay times for YbDD in chloroform at low laser fluence are displayed in Fig. 5a. The $S_{0} \rightarrow S_{2}$ Soret absorption band is shown in orange color, and its stimulated emission band has a small red shift with respect to the ground-state bleach and gives a negative signal ${ }^{49}$. The triplet-triplet $\left(\mathrm{T}_{1} \rightarrow \mathrm{T}_{\mathrm{n}}\right)$ absorption bands are observed at longer wavelengths $(440-530 \mathrm{~nm})^{50}$, with maximum intensity at $451 \mathrm{~nm}$, corresponding to the terminal state energy of $35,578 \mathrm{~cm}^{-1}$. The lifetimes of the bleach and the excited state transients for YbDD were determined by monitoring at wavelengths of 424 and $451 \mathrm{~nm}$, respectively. (Fig. 5b). The two results are effectively the same and are in the picosecond scale, denoting a rapid singlet-to-triplet intersystem crossing. The femtosecond TA absorption spectra were also obtained using a higher pump fluence (Fig. 5c). The pulsed laser with high fluence produces a thermal effect of the YbDD, which causes distortions of the porphyrin structures and results in significant redshifts in the electronic absorption spectra ${ }^{51}$. In contrast to the lower fluence, a redshift of the Soret band $(\Delta \lambda=+$ $36 \mathrm{~nm}$ ) and the $\mathrm{T}_{1} \rightarrow \mathrm{T}_{\mathrm{n}}$ absorption bands were observed. It is worth noting that the structural change was detected instantly by ultrafast TA spectroscopy: the peak at $501 \mathrm{~nm}$ started to shift to $527 \mathrm{~nm}$ after $100 \mathrm{ps}$, and the whole conformation changing process was completed in nanoseconds (Fig. 1d). Furthermore, the formation of the triplet state from the singlet excited state is clearly observed from the kinetics at $527 \mathrm{~nm}$ in Fig. $5 \mathrm{~d}$. In contrast to the $527 \mathrm{~nm}$, the excited singlet state at $501 \mathrm{~nm}$ de-excited exponentially to the ground states. However, when using nanosecond (ns) TA spectroscopy, the detailed kinetics of 

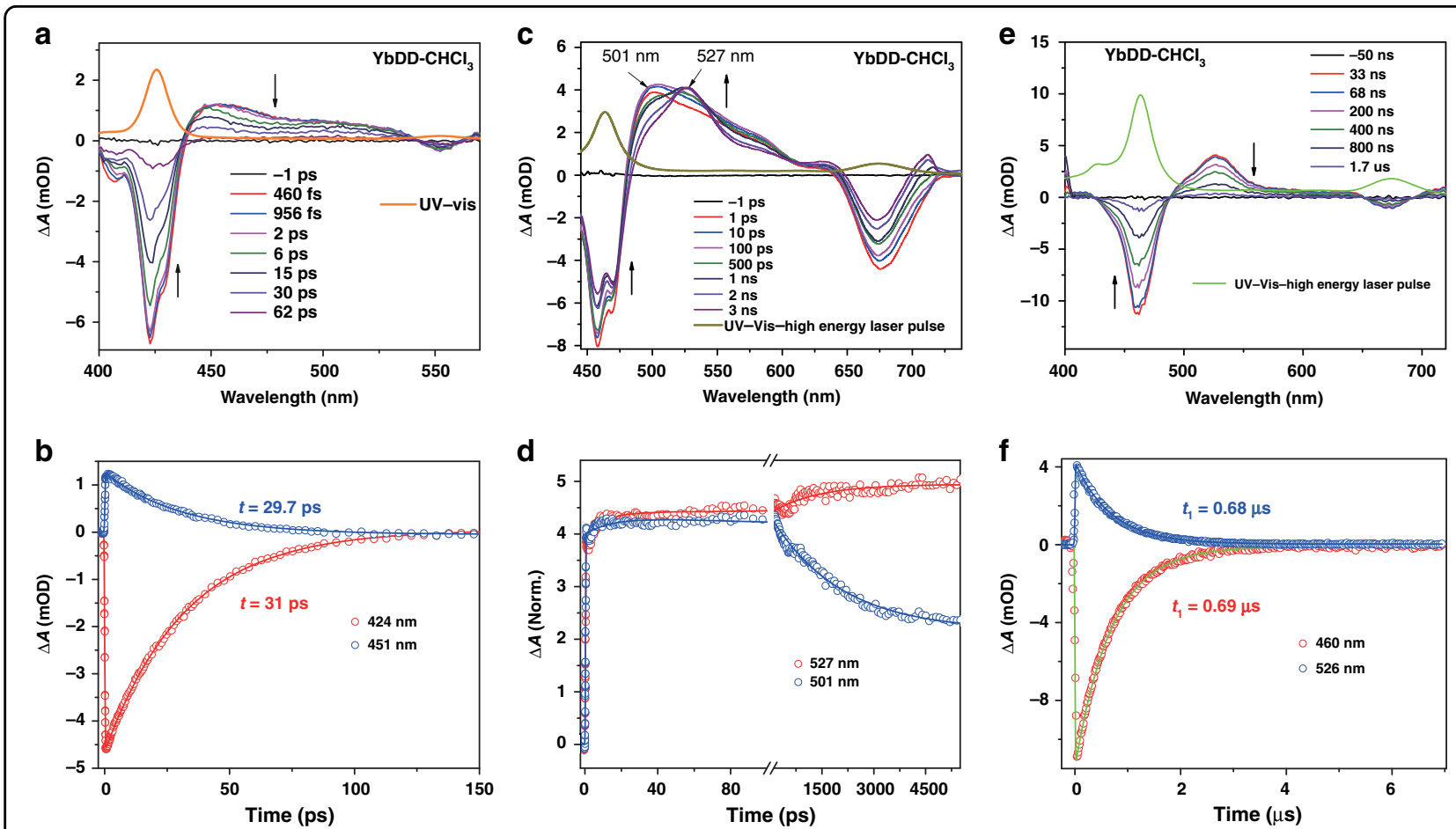

Fig. 5 Transient absorption spectra of YbDD. a fs-TA spectra at different time delays at a low pump fluence $\left(15 \mu \mathrm{J} \mathrm{cm}^{-2}\right)$, $\mathbf{b}$ ground state bleach recovery dynamics at $424 \mathrm{~nm}$ and excited state decay at $451 \mathrm{~nm}$ of YbDD in chloroform following $395 \mathrm{~nm}$ laser excitation. c Spectral evolution in fs-TA at $395 \mathrm{~nm}$ laser excitation having $45 \mu \mathrm{J} \mathrm{cm}{ }^{-2}$ fluence. $\mathbf{d}$ Normalized excited state decay kinetics at 501 and $527 \mathrm{~nm}$. e ns-TA spectra at different time delays with a $45 \mu \mathrm{J} \mathrm{cm}{ }^{-2}$ pump fluence. $\mathbf{f}$ Ground state bleach recovery dynamics at $460 \mathrm{~nm}$ and the excited state decay at $526 \mathrm{~nm}$ of YbDD in chloroform following $395 \mathrm{~nm}$ laser excitation. The UV-vis spectra of YbDD are added in the upper panels to guide the ground state bleach

the triplet-triplet absorption peak at approximately $526 \mathrm{~nm}$ could not be resolved since the conformation was changing too quickly (Fig. 5e). The ground state bleach recovery lifetime was extracted from the nanosecond TA spectra $(0.69 \mu \mathrm{s})$, which is consistent with the decay lifetime by monitoring deactivation of the triplet state signal at $526 \mathrm{~nm}(0.69 \mu \mathrm{s})$ (Fig. 5f). Isosbestic points were found in all TA spectra (Fig. 5a, c, e), which suggest that only one single photoexcited species was formed in each case.

\section{Discussion}

The porphyrin moiety acts as a viable antenna under excitation at $425 \mathrm{~nm}$ for the ytterbium ion. Absorption by the Soret band is followed by an internal conversion cascade to lower singlets and intersystem crossing to $T_{1}$. Calculation shows that there is considerably more than one singlet and one triplet state involved in this cascade. The energy transfer from $\mathrm{T}_{1}$ lifts the $\mathrm{Yb}^{3+}$ ion from the ${ }^{2} \mathrm{~F}_{7 / 2}$ ground state to ${ }^{2} \mathrm{~F}_{5 / 2}$. This change of $\Delta J=1$ is consistent with the first order selection rules for exchange or quadrupole interaction. On the other hand, the donor $\mathrm{T}_{1} \rightarrow \mathrm{S}_{0}$ nonradiative transition is dipole forbidden. Considering the separation between $\mathrm{Yb}^{3+}$ and the porphyrin ring of less than $2 \AA$, the dominant energy transfer mechanism is most likely to be the exchange mechanism.
We report here the first water-soluble lanthanide-porphyrin double-decker complex with structural characterization using NMR, HRMS, STM, and computational chemistry techniques. NIR imaging and cytotoxic ${ }^{1} \mathrm{O}_{2}$ generation of the complexes have also been developed. In our work, the major improvement for bioapplications-considering the low-quantum yield of $\mathrm{Yb}^{3+}$ in the NIR region-comes from the enhancement of the brightness of the potential bioimaging probe YbDD. This property, brightness, has not yet been widely recognized as the yardstick for applicability, compared with quantum yield, but it shows higher practical significance for bioimaging purposes.

\section{Materials and methods General synthesis}

Dichloromethane (DCM), methanol $(\mathrm{MeOH})$ and $\mathrm{n}$ hexanol were dried by refluxing with calcium hydride $\left(\mathrm{CaH}_{2}\right)$ before setting up a reaction. All the chemicals and reagents were of high quality and could be used directly. Reaction processes were monitored by thin-layer chromatography, and further monitored using a UV lamp. Silica gel or $\mathrm{Al}_{2} \mathrm{O}_{3}$ were used for purification in most cases. High-performance liquid chromatography (HPLC) methods were used for final products with high polarity. 
NMR spectra were recorded by either a $400\left({ }^{1} \mathrm{H}\right.$ : $\left.400 \mathrm{MHz},{ }^{13} \mathrm{C}: 100 \mathrm{MHz}\right)$ or a $500\left({ }^{1} \mathrm{H}: 500 \mathrm{MHz},{ }^{13} \mathrm{C}\right.$ : $1250 \mathrm{MHz})$ spectrometer. High-resolution mass spectra were recorded on a Bruker Autoflex II (Bruker Dalton $\mathrm{GmBH}$ ) MALDI-TOF mass spectrometer (characterized by $m / z)$.

\section{Procedures for the preparation of 5,15-bis(3-(2-(2- methoxyethoxy)ethoxy)phenyl)porphyrin (Por-2DEG)}

$\mathrm{Di}(1 \mathrm{H}$-pyrrol-2-yl)methane $(788.84 \mathrm{mg}, 5.4 \mathrm{mmol})$ was dissolved in $1 \mathrm{~L}$ dry DCM in a round flask, and 3-(2-(2methoxyethoxy)ethoxy)benzaldehyde $(1.21 \mathrm{~g}, \quad 5.4 \mathrm{mmol})$ was added to the solution which was stirred for $30 \mathrm{~min}$ under a nitrogen atmosphere to remove oxygen. Next, trifluoroacetic acid $(0.24 \mathrm{~mL}, 3.24 \mathrm{mmol})$ was added slowly. The mixture was stirred at room temperature for $3 \mathrm{~h}$ under a nitrogen atmosphere. After this time, 2,3-dichloro-5,6dicyano-1,4-benzoquinone (DDQ) $(1.47 \mathrm{~g}, 6.48 \mathrm{mmol})$ was added, and the mixture was stirred for an additional $1 \mathrm{~h}$. Then, $2 \mathrm{~mL}$ of triethylamine was added to quench the unreacted TFA. The mixture was stirred for $10 \mathrm{~min}$, and the solvent was removed. The product was purified through silica gel with the solvent gradient DCM: MeOH (100:1).

\section{General procedures for the preparation of $\operatorname{LnDD}(\operatorname{Ln}=\mathrm{Yb}$, $\mathrm{Er}, \mathrm{Gd}$ and $\mathrm{La})$}

5,15-bis(3-(2-(2-methoxyethoxy)ethoxy)phenyl)porphyrin (80.0 mg, $0.12 \mathrm{mmol}), \mathrm{Ln}(\mathrm{acac})_{3} \cdot x \mathrm{H}_{2} \mathrm{O}(0.48 \mathrm{mmol})$, and 1,8 diazabicyclo(5.4.0)undec-7-ene (DBU, $114 \mu \mathrm{L}, 0.79 \mathrm{mmol}$ ) were dissolved in $10 \mathrm{~mL}$ dry hexanol. The mixture was bubbled with nitrogen for $20 \mathrm{~min}$ at room temperature and then refluxed for $12 \mathrm{~h}$ under a nitrogen atmosphere. After reaction completion, the contents were cooled down to room temperature and mixed with $30 \mathrm{~mL}$ hexane. The precipitate was dissolved in DCM and transferred to an $\mathrm{Al}_{2} \mathrm{O}_{3}$ column for purification. (DCM: MeOH 20:1). HPLC was then used for further purification with a preparative column $(\mathrm{C} 18,10.0 \times 250 \mathrm{~mm}, 5 \mu \mathrm{m}$ particle size). The final product was confirmed by MALDI-TOF mass spectral analysis operating in the positive-ion mode using the $\alpha$ cyano-4-hydroxycinnamic acid matrix.

\section{Scanning tunneling microscopy}

An HOPG sample $(10 \times 10 \mathrm{~mm})$ (SPI) was mounted on a Ta plate. The surface was exfoliated with scotch tape and the surface was verified in a UHV Omicron VT-STM at 10-9 mbar. The STM tip (VT-STM Omicron) was Pt/ Ir and was prepared by degassing at $100^{\circ} \mathrm{C}$ for $10 \mathrm{~h}$ and then further cleaned by electron bombardment using a tip preparation tool (Omicron), $2 \mathrm{~A}, 2 \mathrm{~mA}$, and $950 \mathrm{~V}$ for $2 \mathrm{~s}$. In-plane $x-y$ calibration was performed by measuring the atomically resolved HOPG surface lattice parameters. To prepare the monolayer film samples, a droplet $(5-25 \mu \mathrm{L})$ $(1 \mathrm{mmol})$ of a solution in chloroform or toluene was placed in the centre of a clean surface of HOPG and allowed to evaporate at room temperature. The films were dried under roughing vacuum at $10^{-2}$ mbar for $2 \mathrm{~h}$ then transferred to the UHV system for STM imaging.

\section{General spectroscopic characterizations}

The absorption spectra of the final products were measured in aqueous solution in the range $200-800 \mathrm{~nm}$ using an HP Agilent UV-8453 Spectrophotometer. The emission spectra from 400 to $1600 \mathrm{~nm}$ were obtained by the Fluorolog-3 TCSPC (Horiba) combined fluorescence lifetime and steady-state spectrometer. The spectrometer was equipped with an NL-C2 Pulsed Diode Controller NanoLED, which produces picosecond and nanosecond optical pulses at a wide range of wavelengths from the ultraviolet to NIR.

\section{TA spectroscopy}

Helios spectrometers (Ultrafast systems, FL, USA) were used to perform femtosecond transient absorption spectroscopy. The detailed experimental setup of the fs-TA is given in the literature ${ }^{52}$. Briefly, a white-light continuum probe pulse was generated in a 2-mm-thick sapphire plate utilizing a small fraction of the fundamental output of a Ti:sapphire femtosecond regenerative amplifier that was operating at $800 \mathrm{~nm}$ with $35 \mathrm{fs}$ pulses and a repetition rate of $1 \mathrm{kHz}$. Pump pulses at $395 \mathrm{~nm}$ were formed in an optical parametric amplifier (Newport Spectra-Physics). In a 2-mm-thick cuvette cell containing the sample solutions, the pump and probe pulses were overlapped temporally and spatially. The probe light transmitted from the sample was gathered and focused on a broadband $\mathrm{UV}$-visible detector to observe the change in absorbance $(\triangle A)$. The nanosecond TA spectroscopic measurements were also performed at $395 \mathrm{~nm}$ following laser pulse excitation. The ns-TA spectra were recorded using the pump-probe EOS setup (Ultrafast systems, FL, USA), in which a standard probe beam was split into two: one travels through the sample, and the other one is sent directly to the reference spectrometer, which monitors the fluctuations in the probe beam intensity. The detailed experimental setup of the EOS can be found elsewhere ${ }^{53}$.

\footnotetext{
Acknowledgements

This work was supported by the Hong Kong Baptist University (HKBU), Hong Kong Research Grants Council (HKBU 12300117), and the HKBU-HKPolyU Joint Research Program (RC-ICRS/16-17/02). We thank Dr. Zhenyu Liu for his technical support in spectroscopic measurements and Professor Alex Granovsky for communication concerning the use of Firefly.

\section{Author details}

'Department of Chemistry, Hong Kong Baptist University, Kowloon Tong, Hong Kong S.A.R., China. ${ }^{2}$ Hanshan Normal University, Chaozhou, Guangdong Province, China. ${ }^{3}$ Department of Biology, Hong Kong Baptist University, Kowloon Tong, Hong Kong S.A.R., China. ${ }^{4}$ KAUST Solar Center, Division of Physical Science and Engineering, King Abdullah University of Science and Technology (KAUST), Thuwal 23955-6900, Saudi Arabia
} 


\section{Authors contributions}

K.-L.W., W.-K.W. and P.A.T. conceived and supervised the project. P.A.T. performed the calculations. J.-X.Z., W.-L.C. and Y.Z. synthesized the ligands and the complexes. C.X. measured the spectroscopic properties. H.-F.C. performed biological tests. P.M., G.T.H., A.A. and O.F.M. conducted the STM and TAS experiments.

\section{Conflict of interest}

The authors declare that they have no conflict of interest.

Supplementary information is available for this paper at https://doi.org/ 10.1038/s41377-019-0155-9.

Received: 27 December 2018 Revised: 11 April 2019 Accepted: 19 April 2019

Published online: 22 May 2019

\section{References}

1. Kuriki, K., Koike, Y. \& Okamoto, Y. Plastic optical fiber lasers and amplifiers containing lanthanide complexes. Chem. Rev. 102, 2347-2356 (2002).

2. Eliseeva, S. V. \& Bünzli, J. C. G. Lanthanide luminescence for functional materials and bio-sciences. Chem. Soc. Rev. 39, 189-227 (2010).

3. Bünzli, J. C. G. \& Eliseeva, S. V. Lanthanide NIR luminescence for telecommunications, bioanalyses and solar energy conversion. J. Rare Earths $\mathbf{2 8}$, 824-842 (2010).

4. Ye, H. Q. et al. Organo-erbium systems for optical amplification at telecommunications wavelengths. Nat. Mater. 13, 382-386 (2014).

5. Bünzli, J. C. G. Lanthanide luminescence for biomedical analyses and imaging. Chem. Rev. 110, 2729-2755 (2010).

6. Montgomery, C. P. et al. Cell-penetrating metal complex optical probes: targeted and responsive systems based on lanthanide luminescence. Acc. Chem. Res. 42, 925-937 (2009).

7. Armelao, L. et al. Design of luminescent lanthanide complexes: from molecules to highly efficient photo-emitting materials. Coord. Chem. Rev. 254, 487-505 (2010)

8. Beeby, A. et al. Porphyrin sensitization of circularly polarised near-IR lanthanide luminescence: enhanced emission with nucleic acid binding. Chem. Commun. 0, 1183-1184 (2000).

9. Weiss, R. \& Fischer, J. Lanthanide phthalocyanine complexes. In (eds Kadish, K. M., Smith, K. M. \& Guilard, R.) The Porphyrin Handbook. Ch. 105 (San Diego: Academic Press, 2003).

10. Wong, W. K. et al. Synthesis, structure, reactivity and photoluminescence of lanthanide(III) monoporphyrinate complexes. Coord. Chem. Rev. 251 2386-2399 (2007).

11. Lu, G. F. et al. Dysprosium heteroleptic corrole-phthalocyanine triple-decker complexes: synthesis, crystal structure, and electrochemical and magnetic properties. Inorg. Chem. 56, 11503-11512 (2017).

12. Zhao, H. M., Zang, L. X. \& Guo, C. S. Influence of lanthanide ion energy levels on luminescence of corresponding metalloporphyrins. Phys. Chem. Chem. Phys. 19, 7728-7732 (2017).

13. Amokrane, A. et al. Role of $\pi$-radicals in the spin connectivity of clusters and networks of Tb double-decker single molecule magnets. ACS Nano 11, 10750-10760 (2017)

14. Serrano, G. et al. Bilayer of terbium double-decker single-molecule magnets. J. Phys. Chem. C 120, 13581-13586 (2016).

15. He, H. S. Near-infrared emitting lanthanide complexes of porphyrin and BODIPY dyes. Coord. Chem. Rev. 273-274, 87-99 (2014).

16. Bulach, V., Sguerra, F. \& Hosseini, M. W. Porphyrin lanthanide complexes for NIR emission. Coord. Chem. Rev. 256, 1468-1478 (2012).

17. Montalban, A. G. et al. Lanthanide porphyrazine sandwich complexes: synthetic, structural and spectroscopic investigations. J. Chem. Soc. Dalton Trans. $\mathbf{0}$, 3269-3273 (2001).

18. Birin, K. P., Gorbunova, Y. G. \& Tsivadze, A. Y. Selective one-step synthesis of triple-decker (porphyrinato)(phthalocyaninato) early lanthanides: the balance of concurrent processes. Dalton Trans. 40, 11539-11549 (2011).

19. Tarakanova, E. N. et al. Double-decker bis(tetradiazepinoporphyrazinato) rare earth complexes: crucial role of intramolecular hydrogen bonding. Dalton Trans. 45, 12041-12052 (2016).
20. Zang, L. X. et al. Water-soluble gadolinium porphyrin as a multifunctional theranostic agent: Phosphorescence-based oxygen sensing and photosensitivity. Dyes Pigments 142, 465-471 (2017).

21. Peter, H. Inorganic, organometallic and coordination chemistry. In (eds Kadish, K. M., Smith, K. M. \& Guilard, R.) The Porphyrin Handbook. Ch. 18 (San Diego: Academic Press, 2003)

22. Zhang, T. et al. Water-soluble mitochondria-specific ytterbium complex with impressive NIR emission. J. Am. Chem. Soc. 133, 20120-20122 (2011).

23. Zhang, T. et al. Porphyrin-based ytterbium complexes targeting anionic phospholipid membranes as selective biomarkers for cancer cell imaging. Chem. Commun. 49, 7252-7254 (2013).

24. Zhang, T. et al. In vivo selective cancer-tracking gadolinium eradicator as newgeneration photodynamic therapy agent. Proc. Natl. Acad. Sci. USA 111 E5492-E5497 (2014)

25. Ng, D. K. P. \& Jiang, J. Z. Sandwich-type heteroleptic phthalocyaninato and porphyrinato metal complexes. Chem. Soc. Rev. 29, 433-442 (1997).

26. Spyroulias, G. A. \& Coutsolelos, A. G. Evidence of protonated and deprotonated forms of symmetrical and asymmetrical lutetium(III) porphyrin doubledeckers by ${ }^{1} \mathrm{H}-\mathrm{NMR}$ spectroscopy. Inorg. Chem. 35, 1382-1385 (1996).

27. Stewart, J. MOPAC2016. http://OpenMOPAC.net. (2016).

28. Filho, M. A. M. et al. Parameters for the RM1 quantum chemical calculation of complexes of the trications of thulium, ytterbium and lutetium. PLOS ONE 11 e0154500 (2016).

29. Dutra, J. D. L., Bispo, T. D. \& Freire, R. O. LUMPAC lanthanide luminescence software: efficient and user friendly. J. Comput. Chem. 35, 772-775 (2014).

30. Neese, F. The ORCA program system. Wiley Interdiscip. Rev. 2, 73-78 (2012).

31. Granovsky, A. A. Firefly version 8.2.0. http://classic.chem.msu.su/gran/firefly/ index.html. (2013).

32. Schmidt, M. W. et al. General atomic and molecular electronic structure system. J. Comput. Chem. 14, 1347-1363 (1993).

33. Otsuki, J. STM studies on porphyrins. Coord. Chem. Rev. 254, 2311-2341 (2010).

34. Otsuki, J. et al. Arrays of double-decker porphyrins on highly oriented pyrolytic graphite. Langmuir 22, 5708-5715 (2006).

35. Inose, $T$. et al. Switching of single-molecule magnetic properties of $\mathrm{Tb}^{\mathrm{III}}$-porphyrin double-decker complexes and observation of their supramolecular structures on a carbon surface. Chemistry 20, 11237 (2014).

36. Sautet, P. Images of adsorbates with the scanning tunneling microscope: theoretical approaches to the contrast mechanism. Chem. Rev. 97, 1097-1116 (1997).

37. Palmer, R. E. \& Guo, Q. Imaging thin films of organic molecules with the scanning tunnelling microscope. Phys. Chem. Chem. Phys. 4, 4275-4284 (2002).

38. Wang, H. N. et al. Chain-length-adjusted assembly of substituted porphyrins on graphite. Surf. Interface Anal. 32, 266-270 (2001).

39. Foley, T. J. et al. Facile preparation and photophysics of near-infrared luminescent lanthanide(III) monoporphyrinate complexes. Inorg. Chem. 42, 5023-5032 (2003).

40. Tanner, P. A. et al. Misconceptions in electronic energy transfer: bridging the gap between chemistry and physics. Chem. Soc. Rev. 47, 5234-5265 (2018).

41. Liu, Z. H. et al. Brightness calibrates particle size in single particle fluorescence imaging. Opt. Lett. 40, 1242-1245 (2015).

42. Shavaleev, N. M. et al. Influence of symmetry on the luminescence and radiative lifetime of nine-coordinate europium complexes. Inorg. Chem. $\mathbf{5 4}$ 9166-9173 (2015)

43. Luo, S. L. et al. A review of NIR dyes in cancer targeting and imaging. Biomaterials 32, 7127-7138 (2011)

44. Ormond, A. B. \& Freeman, H. S. Dye sensitizers for photodynamic therapy. Materials 6, 817-840 (2013).

45. Malta, O. L. Mechanisms of non-radiative energy transfer involving lanthanide ions revisited. J. Noncryst. Solids 354, 4770-4776 (2008).

46. Werts, M. H. V., Jukes, R. T. F. \& Verhoeven, J. W. The emission spectrum and the radiative lifetime of $\mathrm{Eu}^{3+}$ in luminescent lanthanide complexes. Phys. Chem. Chem. Phys. 4, 1542-1548 (2002)

47. Langlois, A. et al. Metal dependence on the bidirectionality and reversibility of the singlet energy transfer in artificial special pair-containing dyads. Inorg. Chem. 56, 2506-2517 (2017).

48. Liao, M. S., Watts, J. D. \& Huang, M. J. DFT/TDDFT study of lanthanide III monoand bisporphyrin complexes. J. Phys. Chem. A 110, 13089-13098 (2006).

49. Berera, R., Van Grondelle, R. \& Kennis, J. T. M. Ultrafast transient absorption spectroscopy: principles and application to photosynthetic systems. Photosynth. Res. 101, 105-118 (2009). 
50. Masih, D. et al. Photoinduced triplet-state electron transfer of platinum porphyrin: a one-step direct method for sensing iodide with an unprecedented detection limit. J. Mater. Chem. A 3, 6733-6738 (2015).

51. Parusel, A. B. J., Wondimagegn, T. \& Ghosh, A. Do nonplanar porphyrins have red-shifted electronic spectra? A DFT/SCI study and reinvestigation of a recent proposal. J. Am. Chem. Soc. 122, 6371-6374 (2000).
52. Bose, R. et al. Direct femtosecond observation of charge carrier recombination in ternary semiconductor nanocrystals: the effect of composition and shelling. J. Phys. Chem. C 119, 3439-3446 (2015).

53. El-Ballouli, A. O. et al. Quantum confinement-tunable ultrafast charge transfer at the $\mathrm{PbS}$ quantum dot and phenyl- $\mathrm{C}_{61}$-butyric acid methyl ester interface. J. Am. Chem. Soc. 136, 6952-6959 (2014). 